\title{
Analysis of a Channeled Centerbody Supersonic Inlet for F-15B Flight Research
}

\author{
Nalin A. Ratnayake* \\ NASA Dryden Flight Research Center, Edwards, California, 93523, United States
}

\begin{abstract}
The Propulsion Flight Test Fixture at the NASA Dryden Flight Research Center is a unique test platform available for use on the NASA F-15B airplane, tail number 836, as a modular host for a variety of aerodynamics and propulsion research. The first experiment that is to be flown on the test fixture is the Channeled Centerbody Inlet Experiment. The objectives of this project at Dryden are twofold: 1) flight evaluation of an innovative new approach to variable geometry for high-speed inlets, and 2) flight validation of channeled inlet performance prediction by complex computational fluid dynamics codes. The inlet itself is a fixed-geometry version of a mixed-compression, variable-geometry, supersonic inlet developed by TechLand Research, Inc. (North Olmsted, Ohio) to improve the efficiency of supersonic flight at off-nominal conditions. The concept utilizes variable channels in the centerbody section to vary the mass flow of the inlet, enabling efficient operation at a range of flight conditions. This study is particularly concerned with the starting characteristics of the inlet. Computational fluid dynamics studies were shown to align well with analytical predictions, showing the inlet to remain unstarted as designed at the primary test point of Mach 1.5 at an equivalent pressure altitude of 29,500 ft local conditions. Mass-flow-related concerns such as the inlet start problem, as well as inlet efficiency in terms of total pressure loss, are assessed using the flight test geometry.
\end{abstract}

\section{Nomenclature}

$\begin{array}{ll}A^{*} & \text { inlet throat area } \\ A_{n}^{*} & \text { nozzle throat area } \\ A_{1} & \text { inlet capture area } \\ A_{2} & \text { inlet entrance area } \\ K_{S D} & \text { subsonic inlet loss factor } \\ M & \text { Mach number (general) } \\ x & \text { axial distance, in } \\ \text { CCB } & \text { Channeled Centerbody } \\ \text { CCIE } & \text { Channeled Centerbody Inlet Experiment } \\ \text { CFD } & \text { computational fluid dynamics } \\ \text { DFRC } & \text { Dryden Flight Research Center } \\ \text { ECB } & \text { Equivalent Centerbody } \\ \text { NASA } & \text { National Aeronautics and Space Administration } \\ \text { PFTF } & \text { Propulsion Flight Test Fixture } \\ \text { RAGE } & \text { Rake Airflow Gage Experiment } \\ \text { SBIR } & \text { Small Business Innovation Research }\end{array}$

*Aerospace Engineer, Flow Physics Group, Aerodynamics and Propulsion Branch, M/S 4840B, AIAA Senior Member. 


\section{Introduction}

$\mathrm{T}$ HE Propulsion Flight Test Fixture (PFTF) system at the National Aeronautics and Space Administration (NASA) Dryden Flight Research Center (DFRC) (Edwards, California) provides an innovative and cost-effective method of flight-testing advanced propulsion system concepts and components in a relevant environment using an F-15B flight testbed airplane, tail number 836.

The F-15B (The Boeing Company, Chicago, Illinois) is a twoseat trainer version of the F-15 high-performance, supersonic air superiority fighter airplane. It is powered by two Pratt \& Whitney (Hartford, Connecticut) F100-PW-100 afterburning turbofan engines. The PFTF attaches to the centerline pylon of the airplane and has an integrated six-axis force balance for flight-testing propulsion experiments. ${ }^{1}$ A photograph of the F-15B airplane in flight with the PFTF pylon attached is presented as Fig. 1.

The Channeled Centerbody Inlet Experiment (CCIE) is a mixedcompression inlet designed by TechLand Research, Inc. (North Olmsted, Ohio). The CCIE is a fixed-geometry version of the variablegeometry translating channeled centerbody (TCCB), which was designed to address the problem of changing mass flow requirements for supersonic inlets as they pass through different flight conditions. The CCIE is designed to study the operation of the TCCB at offdesign Mach numbers, specifically to assess the mass-flow, pressure recovery, and inlet face distortion of with the channels fully open. Data will be compared against an equivalent smoothed centerbody with no channels but having the same area ratios. A solid model of the CCIE is shown in Fig. 2.

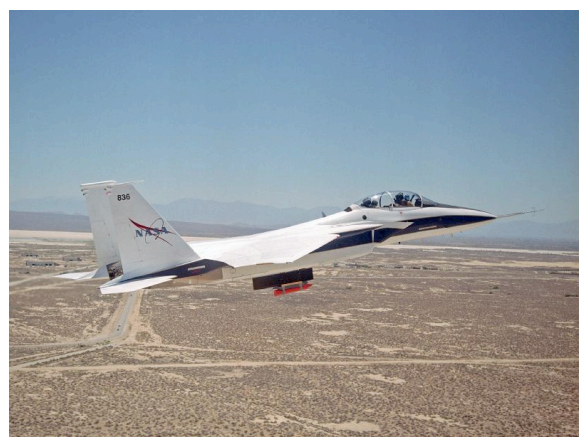

High-speed supersonic inlets typically require designs that accommodate a wide range of throat area; neglecting this in the inlet design leads to extremely inefficient operation at off-design conditions due to improper mass flow ingested by the engine. The CCIE, originally patented by TechLand as the Translating Channeled Centerbody (U.S. Patent Nos. 6,276,632 and 6,705,569), was the result of a Small Business Innovation and Research (SBIR) contract to address flight evaluation for this new approach to variable geometry for high-speed inlets, as well as validation of engine face distortion prediction by complex computational fluid dynamics (CFD) codes. ${ }^{2}$ The translating centerbody inlet designed by TechLand is shown in Figs. 3(a) and 3(b); both images are taken from Ref. 2 .

Though the research is applicable to the general case of variablegeometry supersonic inlets, the CCIE was specifically designed for the Rocket-Based Combined Cycle (RBCC) engine that was under internal NASA development in the early 2000s, and was to even-

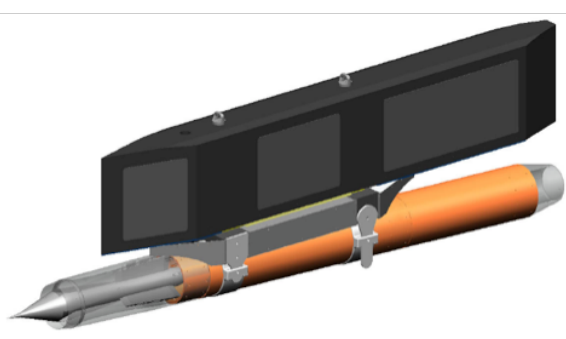
tually be flight-tested at DFRC. ${ }^{3}$ The program was put on hold before completion.

For airbreathing high-supersonic and hypersonic engines, inlets such as the CCIE are expected to provide even greater efficiencies than those already predicted for launch vehicle stages using combined-cycle engines, by allowing for improved performance in the off-nominal, low-supersonic regime. Beyond providing a method of allowing for variable mass-flow (and thus variable duct start/unstart characteristics) in the inlet, the channels also will have two secondary effects on the internal flow: distortion and swirl. Because of the sharp features of the channels, flow distortion and swirl are expected to occur, especially at flight conditions

Figure 2. Solid model of the Channeled Centerbody Inlet Experiment, shown mounted to the Propulsion Flight Test Fixture pylon with duct and throttling nozzle. where the incoming farfield is non-axial. Distortion is generally considered detrimental, as it is associated with increased loss of stagnation (total) pressure. However, particularly for ramjet-type combustors found in combined-cycle engines, swirl can actually increase combustion efficiency via increased mixing. ${ }^{4}$ The tradeoff between these effects is a complex problem and beyond the scope of this paper. 


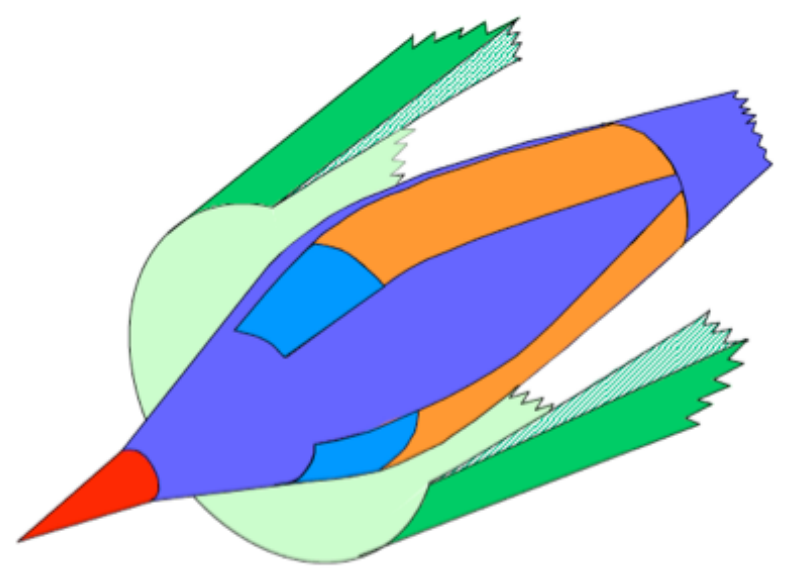

(a) Fully closed configuration.

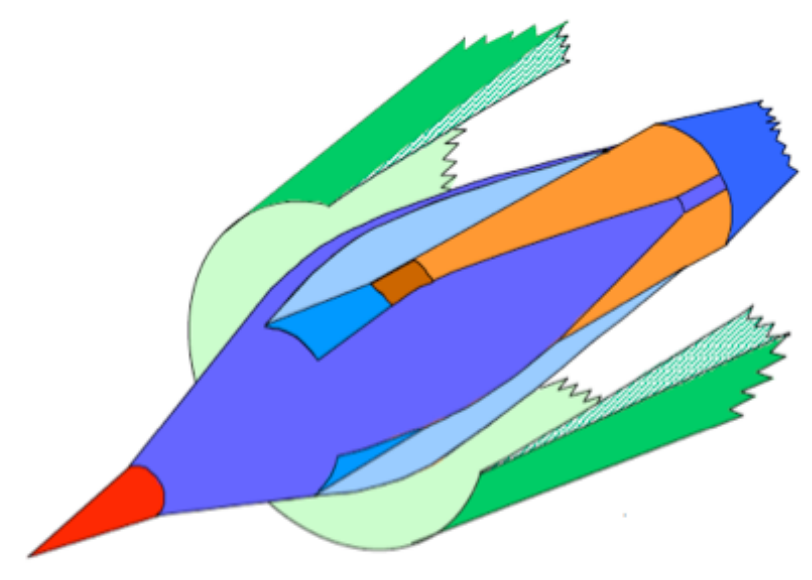

(b) Fully open configuration.

Figure 3. TechLand's translating channeled centerbody inlet; images are taken from Ref. 2.

$$
3 \text { of } 14
$$


The configuration presented for analysis is one of two that will be later flight-tested on the F-15B PFTF. The Channeled Centerbody (CCB) configuration is the CCIE in the specific geometry of being fully actuated (e.g., channels positioned for maximum mass-flow). The configuration analyzed in this study is the Equivalent Centerbody (ECB), a smoothed centerbody that provides the same area distribution as the CCB, but without any channels. Inviscid, viscous/laminar, and viscous/turbulent computations are performed at a range of Mach numbers, and the inlet start curve is assessed.

The inlet starting characteristics are important to know prior to flight-testing, as the CCIE was designed to operate in an unstarted state at an off-design flight condition of Mach 1.5, which will be tested during the planned research flights. The unstarted condition at this Mach number is expected to be stable, which is a positive influence on the safety of the F-15B captive-carry flight tests. Analysis must be conducted, however, to verify this.

TechLand Research, Inc. previously performed extensive axisymmetric and reduced-three-dimensional CFD analysis on the channeled and equivalent centerbody configurations during the design process, including assessments of mass flow, pressure ratio, and distortion. ${ }^{5}$ The present study extends these computational results by using an axisymmetric geometry that is more accurate to the flight hardware.

The geometry definition for this study is shown in Fig. 4, which shows contours as a function of axial distance for the external cowl, the ECB, the CCB channel floor, and the CCB main centerbody. The inlet throat $\left(A^{*}\right)$ and inlet entrance area $\left(A_{2}\right)$ are indicated on the figure. Note that the scale on the vertical axis is stretched; the exaggeration is for clarity in demonstrating the differences between the contours. TechLand's geometries define $\mathrm{x}=0$ as the point where the forebody spike is located when the spike is fully retracted (on the variable-geometry translating channeled centerbody). This off-design configuration has the spike slightly extended, with the spike tip at $\mathrm{x}=-2.035$ inches. Behind the inlet configuration are the duct and throttling nozzle. A plot of the area ratio for both the TechLand and the DFRC configurations, including the duct and throttling nozzle, is shown in Fig. 5. The nozzle throat area $\left(A_{n}^{*}\right)$ is shown for reference.

While the two inlets have similar throat areas (near $\mathrm{x}=13$ inches on the graph), the nature of the contours can be seen to differ between the throat and the aft end of the inlet, and the hardware (DFRC) throat is slightly smaller than the TechLand throat. The differences between the TechLand design and the flight test article form a significant part of the impetus for this study; a model truer to the flight hardware than the TechLand model should be analyzed prior to flight.

The results from this study will be used in the flight test planning process for the CCIE, which at present is planned for flight on the NASA DFRC F-15B, tail number 836, in calendar year 2010.

\section{Theoretical Background and Analytical Calculations}

Analytical techniques for calculating shock location, mass ingestion, and other basic compressible gas dynamic properties of internal flow exist as per several notable references, including an early, definitive work on the subject by Rudolph Hermann, ${ }^{6}$ the classic NACA paper by Kantrowitz and Donaldson, ${ }^{7}$ as well as more modern texts by Anderson ${ }^{8}$ and Mattingly. ${ }^{9}$ Despite the validation of these analytical methods at various specific test points, care should be taken to account for all assumptions made; for example, actual supersonic internal flows (especially in mixed-compression inlets) are quite complex, typically consisting of linked systems of oblique and normal shocks, viscous effects, and also the combined phenomenon of shock/boundary-layer interaction. ${ }^{6}$ These phenomena prompt a healthy degree of caution when applying analytical methods to real inlets.

In general, these classical analytical techniques, including those of Hermann and Kantrowitz, provide a simplified treatment of the detailed flow physics; the mixed-compression scenario that supports a shock train of multiple oblique shocks inside the inlet is typically not considered directly. The core principle is, however, that the inlet throat must be large enough to pass a starting shock, which is a normal shock that sweeps through the inlet with the onset of supersonic flow. The minimum swallowing throat required to pass this shock is always larger than the theoretical (optimum) isentropic De Laval nozzle solution, giving rise to the supersonic inlet starting problem: that the inlet does not start at the isentropic De Laval critical area ratio, but instead requires the throat area to be much larger. The Kantrowitz limit was found to be generally conservative at higher Mach numbers as compared to test data by Van Wie, et al. ${ }^{10}$ By virtue of their mathematical equivalence, it can be concluded that the Kantrowitz limit is similarly conservative in the general case.

It is straightforward to derive from basic flow relations a starting point, the isentropic area-Mach relation. 


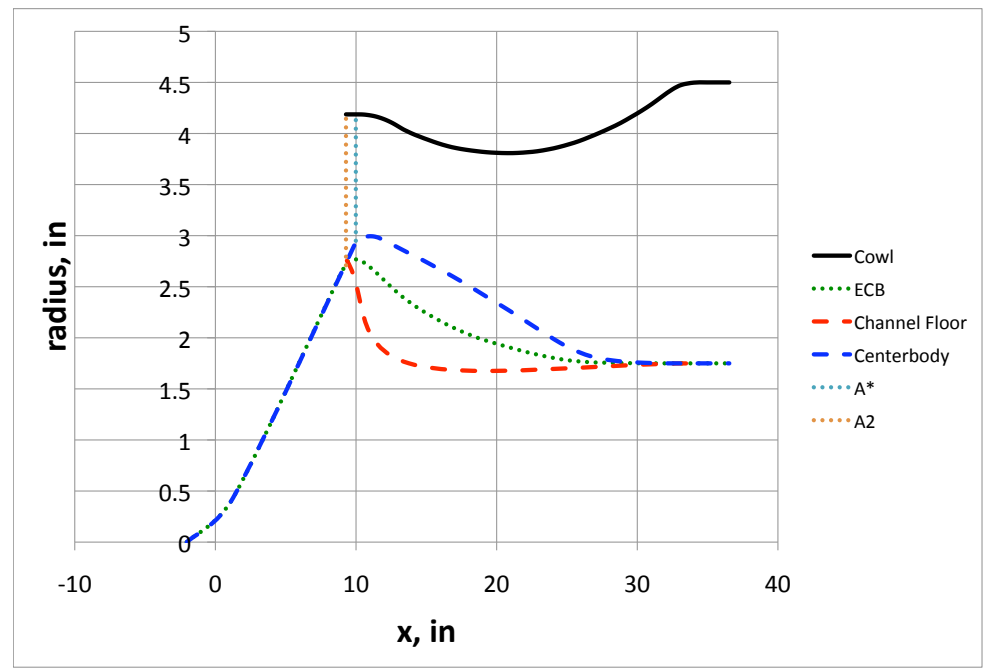

Figure 4. Axial geometry definition for the outer cowl, equivalent centerbody, channeled centerbody, and channel floor.

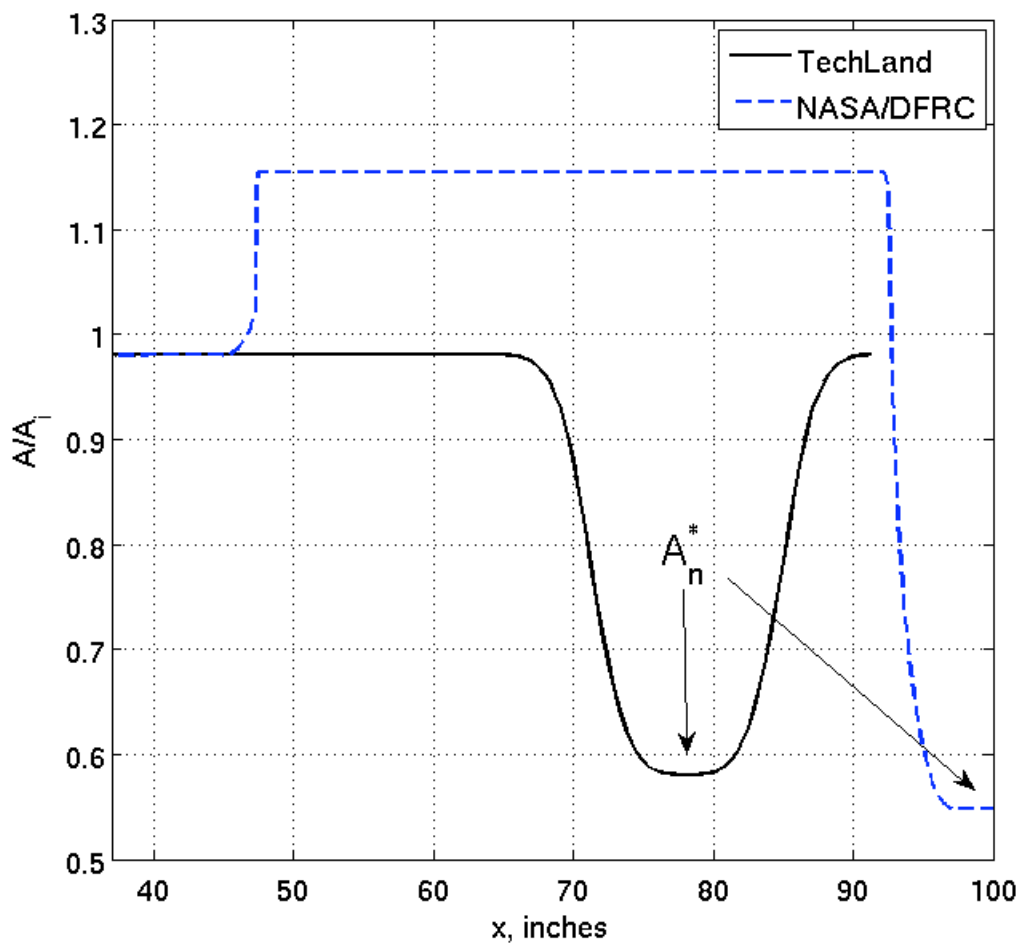

Figure 5. Comparison of the TechLand (equivalent center body) and DFRC (flight) geometries used for CFD. 
Using this equation and the known value of $\frac{A_{1}}{A^{*}}=1.79$, the isentropic limit Mach number for the CCIE was found to be 1.41. It should be noted that this is the freestream Mach number limit, not the inlet entrance Mach number, which is behind two conical shocks and the forebody compression surface.

As derived by multiple sources, one may then arrive at the Kantrowitz Limit by beginning with a variant of the standard area-Mach relation, which provides the mass flow per unit area in a hypothetical duct at a particular Mach number as a simple consequence of conservation of mass. In order to be able to do this, a contraction ratio is typically defined as the inlet entrance area $\left(A_{2}\right)$ over the inlet throat area $\left(A^{*}\right)$.

To account for the starting shock, as the total temperature remains constant over a normal shock (assuming adiabatic flow), the reduction in possible mass flow over such a shock arises solely from a reduction in total pressure; therefore, as shown in Eq. 1, one can take the physical area contraction ratio required by conservation of mass for a sonic throat, and multiply by the total pressure ratio over a normal shock to arrive at Kantrowitz's maximum contraction ratio that permits swallowing of the starting shock:

$$
\left(\frac{A}{A^{*}}\right)_{\text {max }, \text { start }}=\frac{M^{\frac{\gamma}{\gamma-1}-1}\left[\frac{\gamma+1}{\gamma-1}\right]^{\gamma+1 / \gamma-1}\left[1+\frac{\gamma-1}{2}\right]^{-(\gamma+1) / 2(\gamma-1)}}{\left[\frac{2}{\gamma+1}+M^{2}\right]^{\gamma / \gamma-1}\left[\frac{2 \gamma}{\gamma-1} M^{2}-1\right]^{1 / \gamma-1}\left[1+\frac{\gamma-1}{2} M^{2}\right]^{\gamma+1 / 2(\gamma-1)}}
$$

This is mathematically equivalent to the inverse of Hermann's swallowing function, shown in Eq. 2, which describes instead the minimum swallowing-throat as:

$$
\vartheta(M)=\left[\frac{\gamma-1}{\gamma+1}\right]^{1 / 2}\left[\frac{2 \gamma}{\gamma+1}\right]^{1 / \gamma-1}\left[1+\frac{2}{\gamma-1} \frac{1}{M^{2}}\right]^{1 / 2}\left[1-\frac{\gamma-1}{2 \gamma} \frac{1}{M^{2}}\right]^{1 / \gamma-1}=\left[\frac{A^{*}}{A_{2}}\right]_{\text {min, start }}
$$

Both of these functions are general functions of Mach number. For example, by plugging in the Mach number at the entrance to the inlet $\left(M_{2}\right)$ into Eq. 2, one may arrive at the particular ratio $\frac{A^{*}}{A_{2}}$ for the minimum value of throat area $A^{*}$ to swallow the starting shock. These analytical methods may be used to arrive at a baseline rough estimate of the minimum starting Mach number for the CCIE by starting the analysis from the point where flow enters the inlet. This will require estimating the flow behind the two three-dimensional conical shocks generated by the forebody.

The forebody/spike of the CCIE is biconic, which does not lend itself to an easy analytical post-shock solution; however, the average half-angle of the two cones $\left(7.5^{\circ}\right.$ and $\left.8.5^{\circ}\right)$ is $8^{\circ}$. Using this rough estimate of an average right cone, a Taylor-Maccoll solution could be obtained for the flowfield behind the assumed single conical shock. Tabulated results of the Taylor-Maccoll equations and summaries of their significance may be found from a number of sources, one fine example of which is that by Sims. ${ }^{11}$ Using the Mach number on the surface of the cone behind the assumed conical shock as the inlet entrance Mach number, the Kantrowitz limit freestream Mach number for the CCIE inlet was determined from Eq. 1 to be 1.44, which is only very slightly higher than the isentropic limit for this relatively small contraction ratio. This result is the identical result found from Hermann's swallowing function.

While the inlet might now be expected to theoretically start at Mach 1.44, there is an additional factor: the throttling nozzle aft of the duct, which was designed to choke the flow such that the inlet will remain unstarted at Mach numbers well higher than the inlet starting limit. As per Hermann, ${ }^{6}$ the critical nozzle throat area ratio, where the upstream shock first touches the cowl lip may be found from Eq. 3:

$$
\left(\frac{A_{n}^{*}}{A_{2}}\right)=\frac{\vartheta\left(\bar{M}_{2}\right)}{K_{S D}}
$$

where $A_{n}^{*}$ is the critical nozzle throat area, and $K_{S D}$ is a factor that accounts for losses specifically in the subsonic inlet (downstream of the inlet terminal shock). Assuming 5\% losses in the subsonic diffuser downstream of the normal shock, the critical Mach number due to nozzle throttling was determined to be 1.81. This is the expected starting Mach number for the combined system of the inlet and throttling nozzle.

Figure 6 shows the two curves of Kantrowitz limit and isentropic limit versus freestream Mach number, along with the critical Mach number associated with the nozzle throttling ratio. It should be reemphasized, however, that the freestream Mach number is not what was used in the contraction ratio or swallowing function equation in order to generate the curve; the Taylor-Maccoll estimate of the inlet entrance Mach number behind the CCIE forebody, given that particular freestream Mach number, is what was used. For 
example, if the freestream Mach number (x-axis) is 1.5, the Taylor-Maccoll post-shock Mach number along the surface of a 16-degree cone is 1.245. This value is then used to calculate the isentropic and Kantrowitz limits shown in Fig. 6, resulting in curves that are shifted to the right relative to a standard graph showing the curves as direct functions of freestream Mach number.

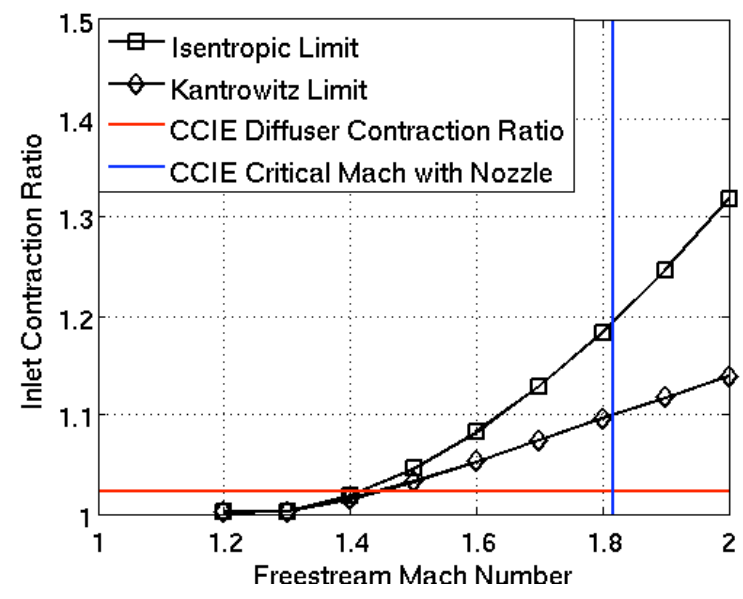

Figure 6. Maximum theoretical ratio of inlet entrance area to throat area that will allow starting, calculated using post-conical-shock Mach number and plotted versus freestream Mach number. bination thereof. The theoretical optimum point to operate the inlet would be with the normal shock swallowed and just aft of the throat. However, any operating point that involves the shock even slightly fore of the throat is an unstable condition, and would result in an inlet unstart.

These analyses were based on many assumptions. The true biconic nature of the CCIE forebody was simplified to a single, average right-circular cone. The average flow Mach number for the inlet entrance was assumed to be equal to the surface Mach number on this average cone, and the inlet entrance was assumed to be inviscid. Spillage effects from the off-nominal flight conditions were not taken into account. Though the distance from the inlet entrance to the throat is only approximately one-half inch, neither the effects of viscosity nor the effects of the oblique shocks that are most certainly present between these two points were considered. Despite this list of simplifications, the analytical calculation provided a reasonable estimate of the more rigorous computational results, as will be demonstrated.

\section{Computational Analysis}

Computational fluid dynamics analysis was performed to extend the analytical predictions and study the problem in more depth. Since the phenomenon of inlet unstart is primarily dependent on area distribution, the CCB unstart characteristics should be at least similar to those of the ECB, which provides for a simpler analysis due to the axisymmetric geometry. The axisymmetric block topology of the ECB is shown with the duct and throttling nozzle (included in this present study) attached in Fig. 7. The grid extends much further downstream (right) and outward (up), but in the interest of showing detail in the CCIE, the image was zoomed in on the area of interest.

Twenty-two interdependent CFD cases were performed using the VULCAN (Viscous Upwind aLgorithm for Complex flow ANalysis) flow solver, developed and maintained by the Hypersonic Airbreathing Propulsion Branch at the NASA Langley Research Center (LaRC). The VULCAN code is a turbulent, non-equilibrium, finite-rate chemical kinetics, Navier-Stokes flow solver for structured, cell-centered, multiblock grids. The 


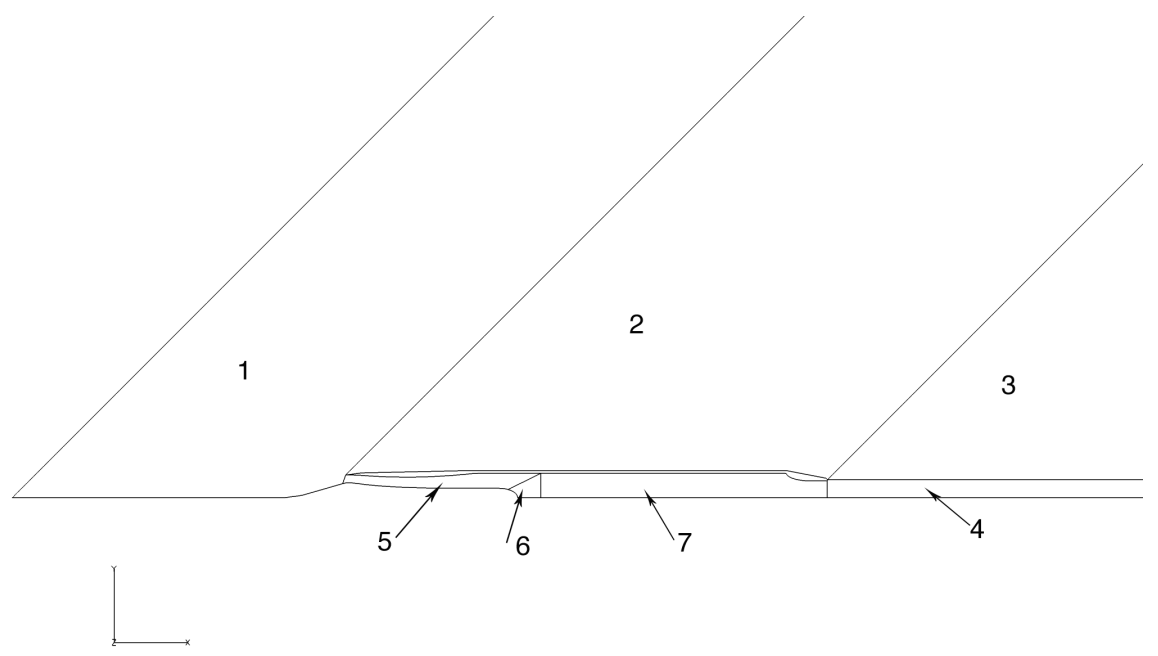

Figure 7. Block topology of the Equivalent Centerbody, shown with the duct and throttling nozzle on the aft end.

computation used a 2nd-Order upwind solver, Van Leer flux limiting, and Edwards low-dissipation fluxsplitting scheme (LDFSS). ${ }^{13}$

Freestream static conditions for the analyses were set to the various Mach numbers at 29,500 $\mathrm{ft}$ pressure altitude (1976 U.S. Standard Atmosphere). A Mach number of 1.5 at this pressure altitude corresponds to the local conditions under the F-15B (behind the forebody shockwaves and inlet effects of the airplane) necessary for the flight experiment. A previous flight program, called the Rake Airflow Gage Experiment (RAGE) helped to determine the local flowfield angularity and in-flight airplane Mach numbers necessary for the desired local flight conditions at the PFTF. The RAGE program is described in Ref. 1.

\section{A. Inviscid Computation}

Cases were run using an Euler solver for Mach numbers 1.0 to 2.0, in increments of 0.1. Care was taken to converge a solution at Mach 1.0 to serve as a starting point. Initially, it was thought that each subsequent solution should be developed incrementally by using the output restart files of the previous Mach number as the initialization flowfield for the next (higher) computation. This was intended to simulate the starting process of the inlet; however, this led to the amplification of what were initially tiny numerical instabilities in each incremental solution. Because this method is essentially a long combined run of a large number of iterations, numerical errors in the solution scheme build cumulatively. Instead of using artificial viscosity or other damping techniques that may adversely influence the solution, the instability problem was addressed by using the converged Mach 1.0 case as a starting point for the Mach 1.1 through Mach 1.5 cases, and the clean Mach 1.5 case as the starting point for the Mach 1.6 through Mach 2.0 cases.

A close-up view of the inviscid grid, showing the inlet portion only, is presented in Fig. 8. The grid is a seven-zone axisymmetric slice of the equivalent centerbody configuration, with the upstream zones aligned with the local Mach wave angle to improve shock capture. The external flow of the inlet is modeled via the outer zones, including the boattail portion of the rear nozzle, providing a more natural exit pressure condition than that of the TechLand study.

Although not physically realistic for the test article and flight condition, the inviscid cases serve as a starting point for the computational analysis, and help to quantify the significance of viscous effects on the internal flow of this and similar inlets. 


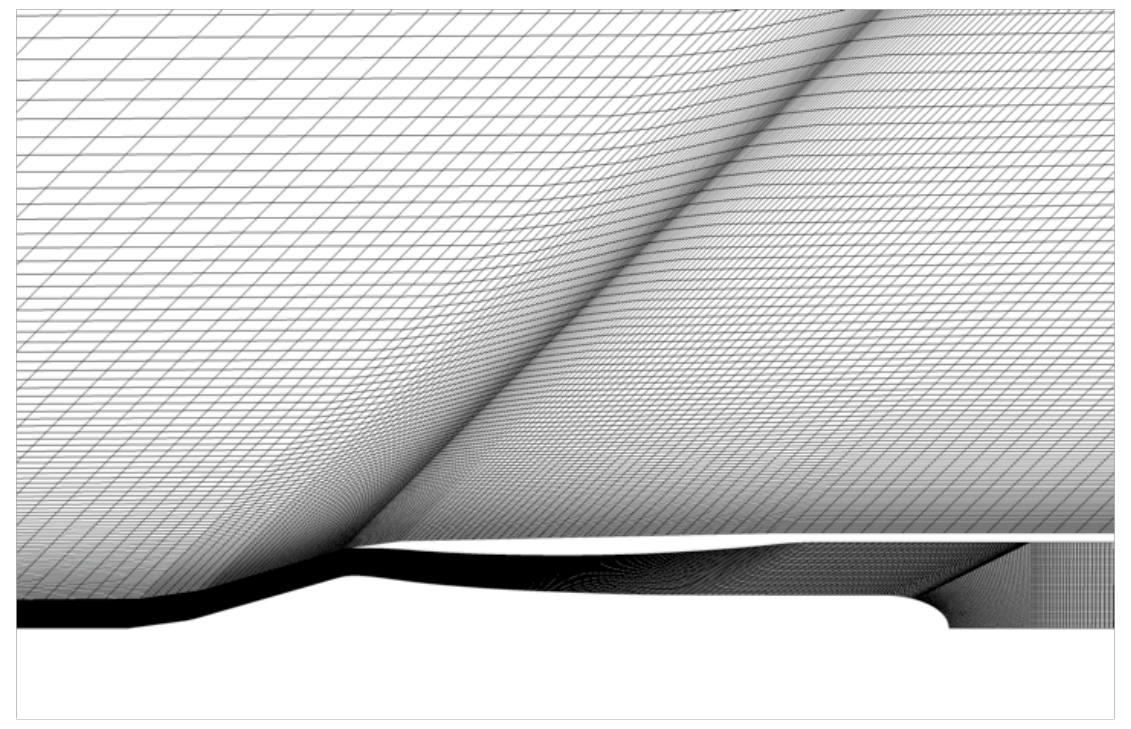

Figure 8. Close-up view of the inlet portion of the inviscid grid.

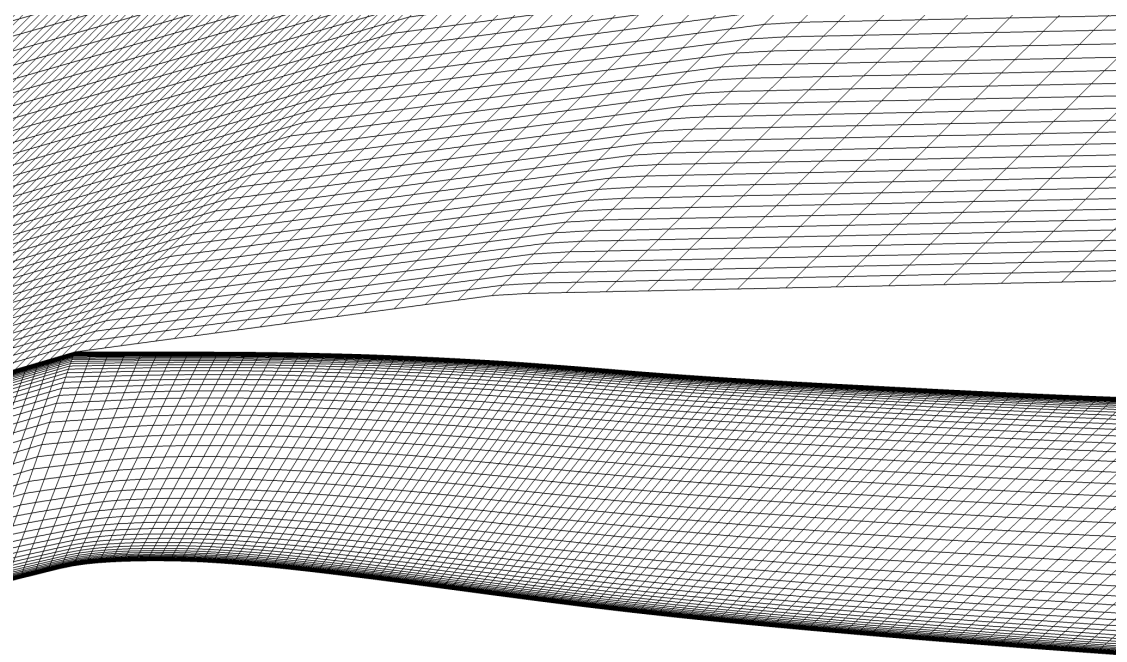

Figure 9. Viscous grid showing the denser mesh near the boundary layer, zoomed in on the entrance portion of the inlet.

9 of 14

American Institute of Aeronautics and Astronautics 


\section{B. Viscous Turbulent Computation}

Once complete, the inviscid runs provided an initial qualitative picture of the flow. The grid was subsequently refined near internal boundaries in order to resolve the boundary layer in the viscous turbulent computations. The external solid boundaries of the cowl, duct, and nozzle were treated as slip walls for computational efficiency. Although this simplification neglected the contribution of skin-friction drag from the outer inlet housing, the present study focuses only on the inlet starting and inlet efficiency. Both of these effects are functions of internal flow and are adequately captured by the present approach.

The turbulent computation was performed using the twoequation Wilcox $k-\omega$ turbulence model. ${ }^{14}$ The turbulence intensity was increased from the default $1.0 \%$ to a level of $2.5 \%$; similarly, the turbulent viscosity ratio was increased from the

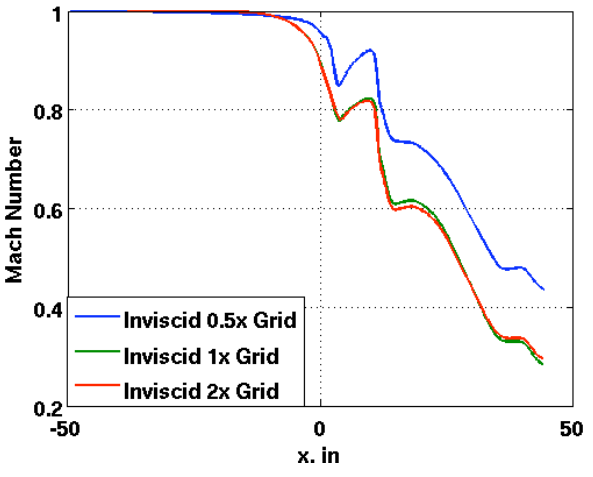

(a) Inviscid grid independence.

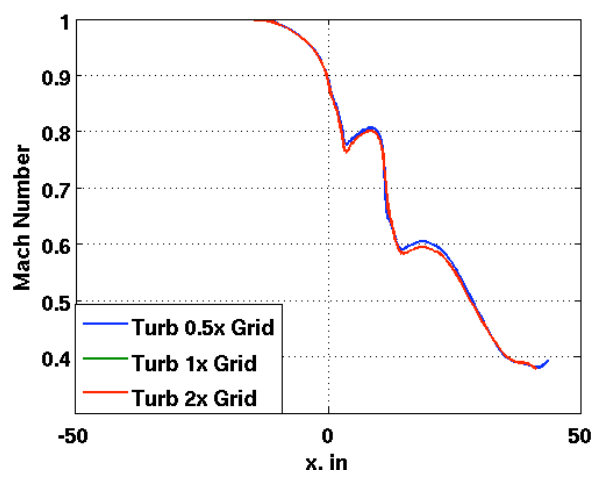

(b) Turbulent grid independence.

Figure 10. Results of grid independence studies on three different mesh sizes. default $10 \%$ to a level of $40 \%$ in the internal zones. Such increases were deemed appropriate for a moderately complex internal flow problem, in which turbulence levels are higher in the flow region of interest than for a purely external flow. These particular values were chosen as the minimum levels required to converge a physically meaningful solution on an initial test case. Convergence and cleanliness of the turbulent results were much improved by restarting the Mach 1.0 turbulent case from a fully converged Mach 0.8 laminar solution, then building incrementally as performed for the inviscid and laminar cases.

The grid used for viscous calculations is shown in Fig. 9. The mesh has been tightened near the walls in order to accurately resolve the boundary layer over an adiabatic, no-slip wall. For the Wilcox $k-\omega$ turbulence model, a y+ value of less than 1 is recommended ${ }^{15}$ the value for this grid is 0.25 , placing approximately 4 points within the laminar sublayer. For the specific case of the $1 \mathrm{X}$ turbulent grid at Mach 1.5, this results in 25 points in the boundary layer at the inlet throat. Each computation was for a steady-state case.

\section{Results}

Grid independence studies were performed for both the inviscid and turbulent grids. Mesh density was doubled in areas of interest, including the forebody compression surface, the complete interior of the inlet-duct-nozzle assembly, and the nozzle plume/wake region. Though total pressure is a parameter of greater interest in the present study, the Mach number in the internal portion of the inlet was chosen as the parameter for grid independence. This is because Mach number, like static pressure, is much more prone to variation across the whole range of the inlet, and thus provides a higher resolution of comparison between two grids.

Comparisons of the Mach number along the compression surface and in the middle of the inlet interior inlet are shown at $0.5 \mathrm{x}, 1 \mathrm{x}$, and $2 \mathrm{x}$ grid densities for the inviscid Mach 1.0 case in Fig. 10(a). In addition, Fig. 10(b) shows a similar comparison for the turbulent Mach 1.0 case. The $1 \mathrm{x}$ and $2 \mathrm{x}$ solutions are quite close; for the inviscid comparison, some minor discrepancies in the curves can be seen. For these cases, the grid independence can be verified by assessing the differences between the two solutions using a flow quantity of interest. The total pressure ratio across the inlet for the $1 \mathrm{x}$ and $2 \mathrm{x}$ inviscid grids differ by less than $0.1 \%$, providing additional assurance of grid convergence. The plots in Fig. 10 are for geometries that were translated from the TechLand reference point such that $\mathrm{x}=0$ inches corresponds to the tip of the forebody spike. 


\section{A. Inlet Start Curves, By Flow Regime}

Figure 11 shows the calculated terminal normal shock location along the longitudinal axis of the geometry as a function of Mach number. Note that the results shown from the CFD cases are with the geometry translated such that $\mathrm{x}=0$ is the CCIE forebody tip. Because the data points shown were taken from the midpoint of the duct, scrutiny was required for points at or near the cowl lip, as the shocks can be bowed or not perpendicular to the axial direction due to inlet geometry, and the inlet throat is less than 1 inch downstream of the cowl. Starting was defined as having no portion of the terminal shock attached to the cowl lip and the midpoint of the terminal shock downstream of the cowl lip. Combining the computational and theoretical solutions, the actual starting limit for the inlet can be reasonably concluded to be somewhere between Mach 1.8 and 1.9. Finer resolution was not deemed necessary, as this is well above the primary CCIE flight test point of Mach 1.5.

It can be seen that the turbulent cases much more closely approximate the Hermann analytical approach (incorporating the throttling nozzle) starting limit than do the inviscid ones. With less momentum and energy loss due to friction in the inte-

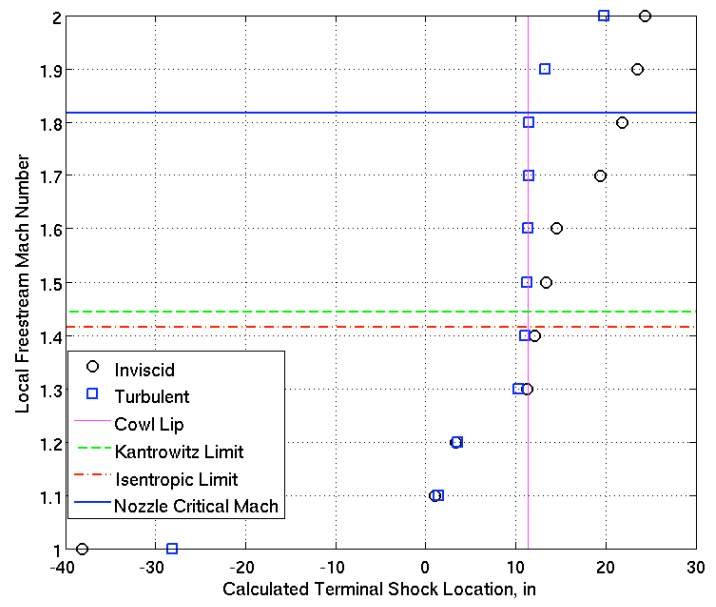
rior, the same geometry in the inviscid run is able to accommodate more mass flow than the turbulent cases, and thus should start well before a realistic Mach number is reached. This is because the growth of the boundary layer in the viscous scenario shrinks the available cross-sectional area of the inlet, making for a smaller effective throat (and consequently a higher effective contraction ratio). The starting Mach number for such flow should intuitively be higher; this supposition is shown by the computational results.

The inviscid runs, however, should not be starting at or below the isentropic limit as indicated by the numerical results. The starting shock that must be passed in order to start the inlet is still a requirement for inviscid flows, and the throttling nozzle is still present in the inviscid geometry. At this time the discrepancy is believed to be a consequence of the extreme difficulty in converging the inviscid solutions for this geometry. Without a turbulence model active, every case above Mach 1.2 exhibited severe separation issues in the diverging portion of the inlet as the flow approached the adverse pressure gradient at the rounded aftbody of the inlet. Further analysis, especially as three-dimensional cases are planned for distortion studies, are expected to clarify the present results.

The turbulent results indicate that the flight experiment should remain safely unstarted, with a solid margin of safety, at the primary local flight condition of Mach 1.5 and an equivalent pressure altitude of 29,500 ft. In planning the CCIE research flights, PFTF/RAGE flight data will be used to correlate the airplane freestream Mach number with the local test conditions at the PFTF pylon location in order to ascertain the appropriate flight condition.

Contours of Mach number for the primary test point (Mach 1.5, unstarted) are shown in Fig. 12(a). A similar contour plot for a started case (Mach 2.0) is shown in Fig. 12(b). It is also apparent from Fig. 12 (b) that the terminal shock is ingested and located at a supercritical position downstream of the throat as expected.

These axisymmetric computational results, while carefully verified with grid independence studies and validated with analytical methods, should still be taken as approximate. While both analytical and computational solutions often do provide a fairly good estimate of the actual values, they need to be validated by flight test. Studies by Van Wie ${ }^{10}$ indicate that there is significant spread in inlet starting Mach numbers of the same contraction ratio, simply due to the variety of geometries and unaccounted-for factors already discussed. Three-dimensional CFD studies of the flight hardware will further quantify inlet starting points, 


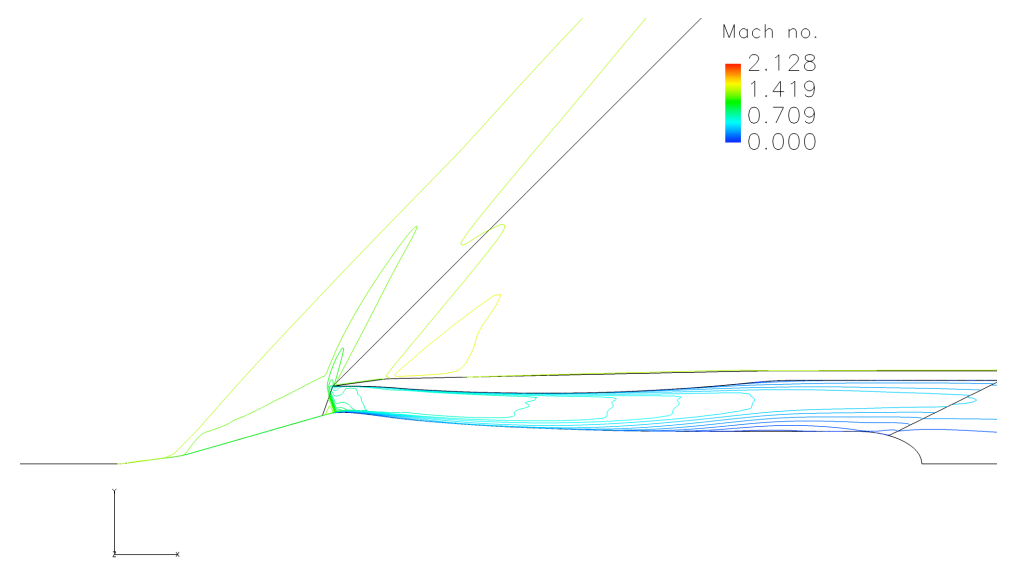

(a) Freestream Mach number $=1.5$.

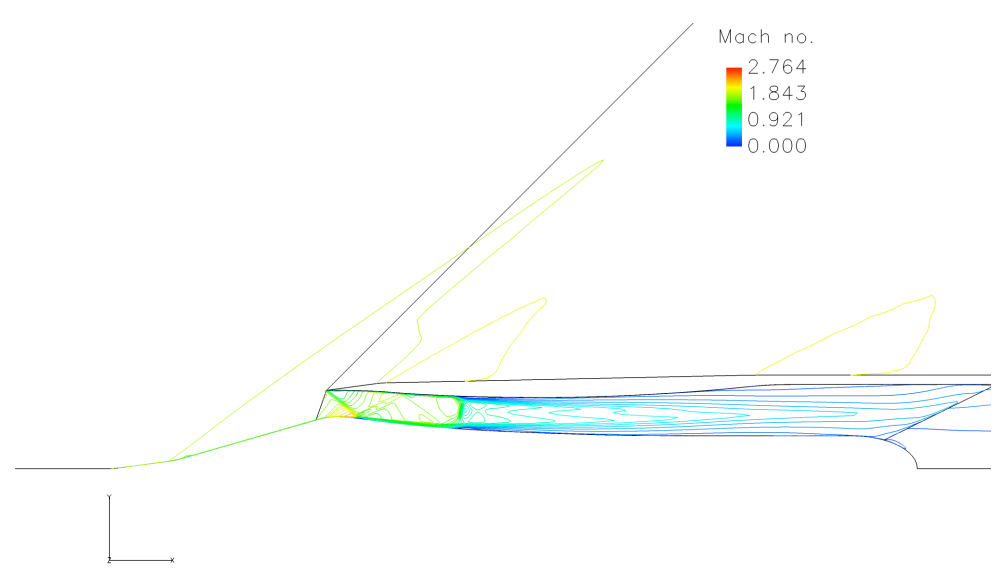

(b) Freestream Mach number $=2.0$

Figure 12. Contours of Mach number for an unstarted and a started case. 
expected mass flow, and distortion levels.

In the case of the ECB, it is reasonable to conclude that additional losses between the entrance and throat for a mixed-compression inlet are not accounted for in the analytical methods designed for pure inlets; the additional flow losses may be increasing the effective contraction ratio, requiring a higher Mach number to start the inlet. These losses would include those from un-modeled internal hardware, such as struts, distortion rakes, et cetera.

\section{B. Inlet Total Pressure Ratio and Mass Flow}

The inlet total pressure ratio, taken from the freestream to the interface plane between the inlet and the duct, is shown as a function of Mach number in Fig. 13(a). The ratio drops with increasing Mach number as expected, as the terminal shock

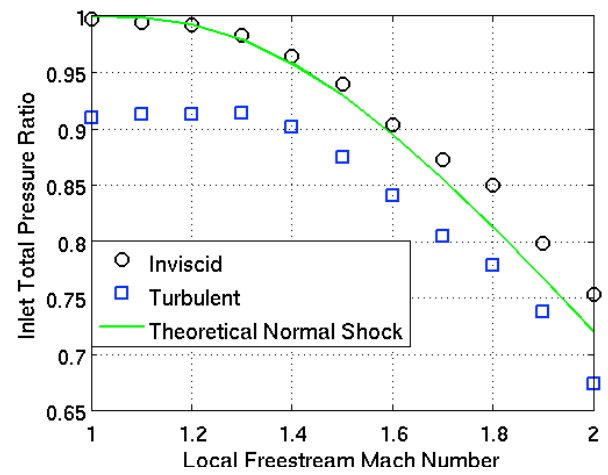

(a) Inlet total pressure ratio as a function of Mach number.

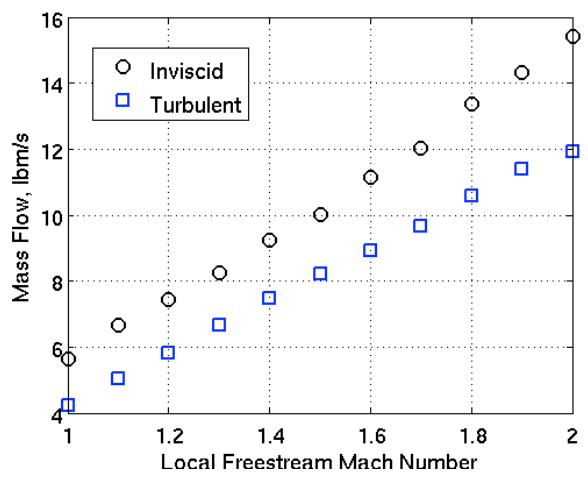

(b) Inlet mass flow as a function of freestream Mach number

Figure 13. Performance of the inlet resulting from inviscid and turbulent cases.

in this paper will be flown on the Propulsion Flight Test Fixture as well and does have the same equivalent area ratios, it is not representative of the Channeled Centerbody geometry. Total pressure losses in the channeled geometry, as well as un-modeled features such as the distortion rakes, struts, and others, may 
make the actual flight articles (both the Channeled Centerbody and Equivalent Centerbody configurations) more restrictive with respect to starting.

\section{B. Future Goals}

Further study will attempt to compare the flight geometry of the Equivalent Centerbody and Channeled Centerbody configurations in a fully three-dimensional computational fluid dynamics analysis, also using the local flowfield flight data. This analysis will attempt to probe the side discrepancies discovered in the present study. A comparison between the two should also quantify the level of distortion introduced by the channels and the associated magnitude of total pressure loss. If later studies are able to also quantify the increase in combustion mixing using the downstream conditions of the inlet modeling presented in this paper, then a tradeoff analysis can be made regarding the value of a) the overall efficiency boost of the propulsion system due to variable-geometry, and b) the increased combustion efficiency on one hand, versus the distortion losses on the other hand. In general, axisymmetric inlets with moderate amounts of internal compression have been shown to offer relatively high total pressure recovery. ${ }^{5}$

Additionally, as the Rake Airflow Gage Experiment flights have shown that on-axial flow at the primary test point may not be possible to achieve in the Channeled Centerbody Inlet Experiment flight tests due to physical constraints on the equipment, off-axial studies of the inlet may be of value. A treatment of inlet buzz and other dynamic effects for safety considerations may also be of operational and academic interest.

Finally, comparing the computational analysis of the three-dimensional configurations to the flight data will provide an important insight into the fidelity of computational fluid dynamics codes in accurately predicting inlet flow distortion in realistic flight conditions.

\section{References}

\footnotetext{
${ }^{1}$ Flynn, D. C., Ratnayake, N. A., and Frederick, M., "Design and Calibration of a Flowfield Survey Rake for Inlet Flight Research," AIAA-2009-1484, 2009.

${ }^{2}$ Weir, L. J., Sanders, B. W., Meleason, E. T., and Podelski, S. D., "Flight Testing of a New Design Concept for Axisymmetric Inlets," TechLand Research Report Number TRR-020406, 2006, Phase II SBIR Final Report, Contract No. NAS4-01031.

${ }^{3}$ Ratnayake, N. A., "Whitepaper on Potential Combined Cycle Engine Research at NASA Dryden," NASA DFRC Internal Memorandum, 2008.

${ }^{4}$ Hill, P. G. and Petersen, C. R., Mechanics and Thermodynamics of Propulsion, 2nd Ed., Addison Wesley Longman, 1992.

${ }^{5}$ Weir, L. J., Sanders, B. W., and Vachon, J., "A New Design Concept for Supersonic Axisymmetric Inlets," AIAA-2002$3775,2002$.

${ }^{6}$ Hermann, R., Supersonic Inlet Diffusers and Introduction to Internal Aerodynamics, Minneapolis-Honeywell Regulator Company, Aeronautical Division, 1956.

${ }^{7}$ Kantrowitz, A. and Donaldson, C. d., "Preliminary Investigation of Supersonic Diffusers," National Advisory Committee for Aeronautics, Advance Confidential Report, ACR-L5D20, 1945.

${ }^{8}$ Anderson, J. D., Modern Compressible Flow, with Historical Perspective, 3rd Ed., McGraw Hill Publishing, 2003.

${ }^{9}$ Mattingly, J. D., Elements of Propulsion: Gas Turbines and Rockets, AIAA Education Series, 2006.

${ }^{10}$ VanWie, D. M., Kwok, F. T., and Walsh, R. F., "Starting characteristics of supersonic inlets," AIAA Paper 96-2914, 1996.

${ }^{11}$ Sims, J. L., "Tables for supersonic flow around right circular cones at zero angle of attack," NASA-SP-3004, 1964.

${ }^{12}$ Barber, T. J., Hiett, D., and Fastenberg, S., "CFD Modeling of the Hypersonic Inlet Starting Problem," AIAA 2006-123, 2006.

${ }^{13}$ Edwards, J. R., "A Low-Diffusion Flux-Splitting Scheme for Navier-Stokes Calculations," Journal of Computers and Fluids, Vol. 27, No. 6, 1995, pp. 635-659.

${ }^{14}$ Wilcox, D. C., "Reassessment of the Scale-Determining Equation for Advanced Turbulence Models," AIAA Journal, vol. 26 (11) pp. 1299-1310, 1988.

${ }^{15}$ Bardina, J. E., Huang, P. G., and Coakley, T. J., "Turbulence Modeling Validation, Testing, and Development," NASA Technical Memorandum 110446, 1997.
} 